\title{
35 années de mobilité scientifique vers l'Allemagne à la MHFA et à l'IFHA
}

\section{Pierre Monnet}

\section{OpenEdition}

\section{Journals}

Édition électronique

URL : http://journals.openedition.org/ifha/417

DOI : 10.4000/ifha.417

ISSN : 2198-8943

\section{Éditeur}

IFRA - Institut franco-allemand (sciences historiques et sociales)

\section{Édition imprimée}

Date de publication : 30 septembre 2012

Pagination : 104-109

ISSN : 2190-0078

\section{Référence électronique}

Pierre Monnet, « 35 années de mobilité scientifique vers l'Allemagne à la MHFA et à l'IFHA », Revue de I'IFHA [En ligne], 4 | 2012, mis en ligne le 14 février 2013, consulté le 25 avril 2019. URL : http:// journals.openedition.org/ifha/417 ; DOI : 10.4000/ifha.417

Ce document a été généré automatiquement le 25 avril 2019

(CIFHA 


\title{
35 années de mobilité scientifique vers l'Allemagne à la MHFA et à l'IFHA
}

\author{
Pierre Monnet
}

1 L'octroi de bourses de mobilité de courte et moyenne durée (de une semaine à un mois par tranche de 150 euros selon la durée et le lieu du séjour, voire davantage en durée et en montant dans le cadre des bourses «Mandrou » et «Monod» ultérieurement créées, respectivement en 2002 et 2006) a constitué dès l'origine de la Mission historique française en Allemagne devenue Institut français d'histoire en Allemagne en 2009 un dispositif constant dans les moyens d'intervention du centre et un axe majeur de sa politique de soutien à la formation d'une jeune recherche française sur l'Allemagne. Ce programme de bourses intervient en complément des deux aides à la mobilité longues, de un à deux ans, réservées depuis 1986 à des doctorants résidant cette fois en poste dans l'institut.

2 Le temps nous a semblé venu de proposer un bilan quantitatif et qualitatif de ce dispositif qui, en dépit des changements intervenus dans le paysage universitaire français et des mutations qu'ont connues bien des institutions scientifiques franco-allemandes, continue de susciter l'intérêt de nombreux candidats. Ce bref inventaire rassemble quelques données statistiques glanées dans les rapports d'activités et les numéros du Bulletin puis de la Revue de notre institution, suivies d'un témoignage porté par un bénéficiaire actuel de ces bourses sur leur utilité et leur valeur ajoutée dans la construction d'un parcours de recherche.

Le fonctionnement et l'attribution de ces bourses n'ont jamais varié dans leurs principes depuis 35 ans. Dès l'origine il s'est agi d'un appel d'offres permanent, léger et flexible (consultable aujourd'hui sur le site internet). Contrairement en effet à la pratique établie dans de nombreux autres centres de recherche ou agences scientifiques, il n'existe pas de délais ou de sessions fixes pour le dépôt d'une demande. Les candidats peuvent ainsi s'adresser à tout moment de l'année à l'IFHA, qui traite les dossiers au fil de l'eau et dans le respect des marges budgétaires attribuées par le Ministère des Affaires Étrangères pour financer cette action. Le temps de réaction entre le besoin exprimé d'une mobilité brève 
et son éventuel accord ne dépasse jamais deux à quatre semaines. Cette souplesse permet donc aux intéressés de concevoir une mobilité scientifique vers l'Allemagne, sans restriction géographique comme en témoigne la carte des 60 destinations répertoriées et qui couvre l'ensemble de l'espace germanique y compris autrichien, au plus près de leur rythme de travail et de leurs besoins afin d'effectuer un séjour en archives ou en bibliothèques au gré des vacances universitaires, mais aussi d'un séjour prolongé avant ou après un colloque, mais encore d'une lacune documentaire brusquement apparue au moment de la vérification des informations ou bien enfin de la rédaction d'un mémoire, d'une thèse, d'un article ou d'un livre. De la sorte, le dispositif des bourses de brève durée ne vient pas concurrencer mais bien plutôt compléter les programmes de mobilité mis en place par d'autres organismes, souvent mieux dotés mais reposant sur une exigence, légitime dans leurs cas, d'une prévision de séjour à plus long terme, qu'il s'agisse du CNRS, du DAAD, de la DFG, de l'OFAJ, du CIERA etc., qu'il s'agisse encore des allocations de recherche des écoles doctorales, de celles du Centre Marc Bloch de Berlin, des collèges doctoraux franco-allemands, sans même parler des bourses des fondations allemandes, ou des cursus plus structurés de l'Université franco-allemande et des séjours longs dans le cadre des programmes Erasmus, Erasmus Mundus, Procope...

4 Il convient d'ailleurs de noter la belle constance et l'admirable résistance du dispositif des bourses de l'IFHA aux changements profonds qu'introduisirent tour à tour et depuis trois décennies la mise en place du programme Erasmus, le processus de Bologne, la mastérisation des études et des concours, l'articulation entre allocations doctorales et écoles doctorales, la création de nouvelles structures et agences scientifiques francoallemandes...

5 Au total, depuis l'implantation de la MHFA à Göttingen en 1977 puis son transfert à Francfort en 2009, ce sont 843 étudiants de maîtrise et DEA (comptabilisés en master 1 et 2 depuis 2005), doctorants et post-doctorants qui ont bénéficié d'une bourse de mobilité. Reporté sur les 35 années de fonctionnement du dispositif, ce chiffre aboutit à une moyenne de 25 bourses distribuées par an. Toutefois, cette moyenne ne vaut véritablement qu'à compter du début des années 1990 : auparavant en effet, de 1977 à 1992, 125 bourses avaient été accordées, soit une moyenne de 8 bénéficiaires par an, ce chiffre correspondant à la phase de démarrage budgétaire et administratif d'un centre encore en construction et en croissance. Cette moyenne de 25 bourses sur près de 35 ans a cependant connu des fluctuations, évoluant entre un étiage bas de quelque 25-30 en 1997, 2007, 2009, 2010 et 2011, et une fourchette haute de 40-50 par an en 1998, 2000, 2001, $2002,2003,2004,2005,2006$ et 2008. Le record absolu se situe en 2000 avec un nombre de 70 bourses accordées. Le premier bilan statistique tiré de ce programme à l'occasion des 20 ans de la MHFA célébrés en 1997 avait révélé que pour la période 1984-1997, pendant laquelle un tel décompte était possible, 195 boursiers historiens avaient bénéficié de ce soutien, 59 inscrits en maitrise, 91 en DEA et doctorat et 43 pour des recherches postdoctorales.

6 Pour la seconde tranche de 15 ans de 1997 à 2012, les bénéficiaires des bourses étaient pour 109 d'entre eux inscrits en maîtrise (sans le DEA devenu master 2 en 2005 dans la comptabilité de la MHFA), pour 266 en doctorat (avec les DEA jusqu'en 2005) tandis que 214 poursuivaient des recherches postdoctorales. D'une période de 15 ans à l'autre, la proportion d'étudiants de maitrise est toujours demeurée de moitié inférieure (60\% avant 1997 et $40 \%$ après 1997) à celle des doctorants, tandis que la proportion des postdoctorants connaissait après 1997 un saut quantitatif spectaculaire. La proportion des 
étudiants de maîtrise sur toutes ces années s'est toujours située grossièrement entre un quart et au mieux un tiers des effectifs, un taux qui s'explique par le fait que le choix d'un sujet d'études germaniques intervient rarement dès la première année du mémoire et que la consultation d'archives ou le séjour en bibliothèque déclenche souvent une demande de bourse déposée auprès du centre dans une phase avancée de la recherche, peu avant la soutenance ou bien auparavant au niveau du DEA et désormais au cours du master 2 bien plus qu'au cours de la maitrise puis du master 1. Parallèlement, la proportion des étudiants inscrits en doctorat est toujours demeurée la plus forte, avant comme après 1997, représentant régulièrement entre $50 \%$ et $60 \%$ des effectifs. C'est en effet à ce niveau d'études et de recherche que le dispositif des bourses de la MHFA puis de l'IFHA s'est révélé et continue de se manifester comme le plus pertinent. Cette situation s'explique non seulement par la stabilité du sujet de recherche alors conduit sur plusieurs années, mais aussi par la bonne connaissance dont les directeurs de thèse disposent de cet instrument de soutien et par le bon usage qu'ils en font, mettant au jour l'existence d'un actif et solide réseau de relais et d'orientation au sein de l'institution universitaire française. Justement, et ce n'est pas là le moindre bénéfice du dispositif, ce réseau de collègues français spécialisés en histoire allemande et franco-allemande n'a cessé depuis une trentaine d'années d'être en augmentation, ce dont témoigne la proportion de plus en plus importante de bénéficiaires des bourses du centre au niveau postdoctoral. La MHFA puis l'IFHA ont donc joué, notamment grâce au système des bourses de courte durée, un rôle majeur dans la formation d'une nouvelle génération de jeunes chercheurs, désormais en poste, sur l'Allemagne, et capables aujourd'hui d'encadrer les travaux de jeunes étudiants faisant à leur tout appel aux aides à la mobilité scientifique courte vers l'Allemagne. Pour la seule période 1990-1997, les bénéficiaires des bourses de la MHFA avaient soutenu dans la foulée 4 thèses d'État, 3 habilitations à diriger des recherches et 40 doctorats. La situation n'est pas différente pour la décennie 2000-2010, ce que confirme également l'existence, parmi cette même population, d'un vivier fidèle de contributeurs et de recenseurs activement impliqués dans la production de la Revue du centre.

Quant au champ chronologique couvert par les recherches de ces boursiers, à plus de $90 \%$ des historiens, le bilan statistique dressé en 1997 à l'occasion des 20 ans de la MFHA faisait état de la répartition par période suivante: $36 \%$ des sujets soutenus relevaient de l'histoire médiévale, $32 \%$ de l'histoire moderne et $29,5 \%$ de l'histoire contemporaine. Pour les années 1997 à 2012, la distribution chronologique des 593 sujets soutenus donne les résultats suivants : 240 en histoire médiévale ( $40 \%), 204$ en histoire moderne (34\%) et 149 en histoire contemporaine (25\%).

8 Au total, ni le déménagement de la MHFA de Göttingen à Francfort en 2009, ni l'offre parallèle de bourses proposées par d'autres organismes n'ont porté préjudice au déploiement du dispositif ancien et souple de bourses mis en place depuis plus de 30 ans par le centre. Elles demeurent une possibilité utilisée à tous les niveaux de la recherche, pour des durées variables et couvrant la large palette du temps long des études historiques, y compris en histoire contemporaine. De même, et en dépit d'une concentration légitime des séjours dans de grands pôles muséaux, universitaires, documentaires et scientifiques tels que Berlin, Munich, Francfort, Cologne, Wolfenbüttel, Göttingen (y compris après 2009), Stuttgart, Nuremberg ou Vienne, le système permet aux jeunes chercheurs de rayonner dans tout l'espace académique allemand et germanophone. 
9 Au terme de ce bref inventaire, un seul motif d'inquiétude ou plutôt un seul souhait subsiste, en dehors du maintien de l'effort budgétaire pour financer ce programme, celui de ne pas assister à une sédentarisation trop forte des étudiants à la faveur d'un alourdissement des maquettes et modules d'enseignement en master, des doctorants au regard de la structuration des parcours de formation doctorale et des collègues à la suite de l'alourdissement continu des charges administratives et budgétaires pesant sur leur emploi du temps. La mobilité physique, mais surtout l'appétit à bouger et à circuler demeurent en effet non seulement la condition indispensable à toute recherche innovante mais aussi la justification d'un programme de bourses que l'IFHA continuera dans toute la mesure de ses moyens à faire figurer parmi les priorités de son action.

\section{ANNEXES}

\section{La campagne 2012 des bourses d'été Monod et Mandrou : un bon cru}

Comme chaque année, l'IFHA a proposé en 2012 deux bourses d'été pour permettre à un chercheur postdoctorant (bourse Monod) et à un doctorant (bourse Mandrou) d'effectuer une mobilité scientifique en Allemagne d'une durée de 4 à 8 semaines.

23 dossiers au total ont été adressés à l'IFHA, 6 pour la bourse Monod et 17 pour la bourse Mandrou. Du côté des jeunes chercheurs candidats à la bourse Monod (dont la moitié est originaire de Paris et les trois autres de Lille, Nancy et Strasbourg), la période contemporaine avec 4 dossiers concentre les recherches, tandis que deux modernistes avaient fait acte de candidature, la période médiévale ne se trouvant pas représentée cette année. Sur les 6 sujets, 3 relevaient clairement de l'histoire politique (tous 3 d'ailleurs en contemporaine), 2 de l'histoire culturelle (en histoire moderne) et 1 en histoire économique. En 2012, c'est un dossier d'histoire moderne qui a été retenu, deux autres dossiers ayant été convertis en bourse de courte durée et deux n'ayant pas été retenus après la sélection du comité scientifique.

Pour les doctorants candidats à la bourse Mandrou, sur 17 dossiers 7 n'ont pas été retenus (soit un taux d'échec de $40 \%$ comparable à celui de la bouse Monod) et 9 autres ont été convertis en bourse de courte durée, tandis que la bourse Monod était attribuée à un doctorat en histoire contemporaine. C'est d'ailleurs cette dernière période qui avec 11 dossiers se taille la part du lion, les autres se répartissant entre l'histoire moderne (4) et l'histoire médiévale (2). Au sein de la période contemporaine, la majorité des sujets relève de l'histoire politique ou diplomatique (6 sur 11). Dans l'ensemble, comme c'était déjà le cas pour la bourse Monod, l'histoire économique avec 2 dossiers demeure le parent pauvre. Parmi ces dossiers de doctorants, on remarquera la part conséquente occupée par les cotutelles ( 5 sur 17), une proportion qui confirme la lente mais certaine progression de ce mécanisme, particulièrement dans le domaine franco-allemand.

Le jury de l'IFHA a été confronté à la difficulté non pas d'attribuer les deux bourses mais de choisir parmi les bons dossiers, preuve de l'attractivité affirmée du dispositif des 
bourses en dépit d'une concurrence importante compte tenu du nombre d'aides proposées par de nombreuses autres institutions.

\section{AUTEUR}

\section{PIERRE MONNET}

Pierre Monnet est directeur de l'IFHA. 